\title{
Core Evolution of Rich Stellar Clusters in the LMC
}

\author{
A. D. Mackey, M. I. Wilkinson, \& G. F. Gilmore \\ Institute of Astronomy, Madingley Road, Cambridge, CB3 OHA, UK
}

J. R. Hurley

American Museum of Natural History, New York

\begin{abstract}
Observations of rich $\mathrm{LMC}$ clusters reveal an increasing spread in core radius with increasing age. We use $N$-body simulations of clusters on elliptical orbits about the LMC to show that a time-varying tidal field can result in significant core expansion. The observed spread in core radii may therefore reflect a spread in orbital parameters between clusters.
\end{abstract}

\section{The Core-radius vs. Age Relationship}

As part of a detailed study of stellar clusters in the LMC we have compiled and reduced two-colour data from the HST archive for 47 rich LMC clusters and determined a set of structural parameters for the sample (Mackey \& Gilmore 2001). If core radius is plotted against age, Figure 1(a), an evolutionary trend is revealed - namely that the spread in core radius increases significantly with age. This relationship has previously been noted and studied by Elson and collaborators (Elson, Freeman, \& Lauer 1989; Elson 1991; Elson 1992). Both a cluster's IMF and binary star fraction influence the evolution of its core; however, our HST data and $N$-body models suggest neither is the dominant mechanism in producing the observed spread in core radius (Wilkinson, et al. 2001). There is a third possibility - a time-varying tidal field could affect the rate of core expansion in a cluster over an appropriate time-scale. The observed spread in core radii would then reflect a spread in orbital parameters between clusters.

\section{2. $\quad N$-body Simulations}

We simulate clusters of 5000 stars using Aarseth's NBODY4 direct $N$-body code with stellar and binary evolution algorithms running on the HARP special purpose computer and modified to include the effects of time-dependent tidal fields. As a starting point we study the evolution of clusters placed on elliptical orbits about a point mass LMC. Each model cluster starts in virial equilibrium with an isotropic velocity distribution, a Plummer density profile, metallicity $Z=0.02$, a Kroupa, Tout, \& Gilmore (1993) IMF and no primordial binaries (so far). The core radius is estimated according to the definitions of Casertano \& Hut (1985).

Figure 1(b) shows a comparison in the core evolution of clusters on circular and eccentric orbits. To show clearly here the possible effects of a time-varying 

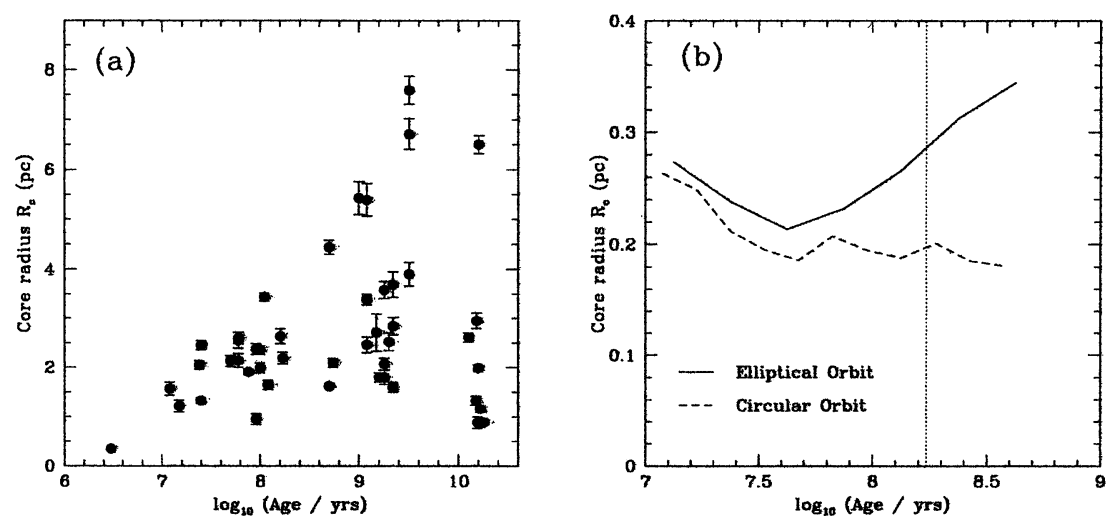

Figure 1. Core radius vs. age for (a) our observational sample and (b) simulated clusters - each curve represents the average of several runs. The dotted line marks peri-LMC approach.

tidal field we take an extreme case - clusters on an orbit with ellipticity 0.6 and apo-LMC distance $8 \mathrm{kpc}$, and on a circular orbit, radius $2 \mathrm{kpc}$. The evolution of core radius is very different between the two cases, with clusters on the eccentric orbit undergoing significant core expansion due to spending longer times at radii where the tidal field is weaker. We do not get radii as large as those of rich LMC clusters because of the small size of our simulated clusters. We emphasize that the case of an elliptical orbit has been used simply to illustrate the possible effects of a time-varying tidal field - LMC clusters are usually assumed to be on circular orbits. However, it is important to note that the LMC and SMC form a binary pair of galaxies with close encounters every few $\times 10^{8}$ years $(\mathrm{eg}$ Zhao 1998). Such a close encounter could easily cause a significant spread in the eccentricities of the older LMC clusters. A GRAPE-6 computer is now available to us, allowing us to simulate clusters of $N \sim 50000$ stars (a similar mass to many clusters in the observational sample) and so verify our results and explore further the effects of time-varying tidal fields.

\section{References}

Casertano, S. \& Hut, P. 1985, ApJ, 298, 80

Elson, R. A. W. 1991, ApJS, 76, 185

Elson, R. A. W. 1992, MNRAS, 256, 515

Elson, R. A. W., Freeman, K. C., \& Lauer, T. R. 1989, ApJ, 347, L69

Kroupa, P., Tout, C. A., \& Gilmore, G. F. 1993, MNRAS, 262, 545

Mackey, A. D., \& Gilmore, G. F. 2001, in preparation

Wilkinson, M. I., Hurley, J. R., Mackey, A. D., \& Gilmore, G. F. 2001, in preparation

Zhao, H. 1998, ApJ, 500, L149 